\title{
El pensador bajo la máscara. Aporías a la filosofía experimental
}

Osman Daniel Choque Aliaga

Albert-Ludwigs-Universität Freiburg, Alemania 


\title{
El pensador bajo la máscara. Aporías a la filosofía experimental*
}

Resumen: el pensador suizo Andreas Urs Sommer es, sin dudarlo, uno de los actuales especialistas de Nietzsche. En el año 2017 publica un texto titulado Nietzsche und die Folgen, un libro que recobra la figura del pensador alemán a la luz de ideas bastantes novedosas que hasta ahora no habían sido presentadas por la mayoría de los intérpretes de Nietzsche. En ese sentido, la filosofía experimental (Experimentalphilosophie) que presenta Sommer es la que ha llamado la atención de la crítica. Se trataría de esconder bajo el juego experimental la filosofía de Nietzsche, omitiendo de ese modo un cierto tipo de compromiso de sus postulados filosóficos. Esta determinación está cobrando fuerza en la medida en que nos muestra las consecuencias de la filosofía experimental. Este trabajo pretende desarrollar, por un lado, los postulados centrales de la propuesta de Sommer para luego confrontarlos a la luz de la crítica y en qué medida dicha crítica es medular. Lo anterior da luces a una discusión que, más que inclinarse a la polémica, muestra el nutriente de una fructífera investigación.

Palabras clave: Nietzsche, Sommer, filosofía experimental, Zaratustra.

\section{The thinker under the mask. Aporias to the experimental philosophy}

\begin{abstract}
Swiss thinker Andreas Urs Sommer is, undoubtedly, one of Nietzsche's current specialists. In the year of 2017 publishes a text entitled Nietzsche und die Folgen, a book that recovers the figure of the German thinker in the light of quite novel ideas that until now had not been presented by most of the interpreters of Nietzsche. In that sense, the experimental philosophy (Experimentalphilosophie) presented by Sommer is the one that has caught the attention of critics. It would be a matter of hiding Nietzsche's philosophy under the experimental game, thus omitting a certain type of commitment from his philosophical postulates. This determination is gaining strength insofar as it shows us the consequences of experimental philosophy. This work aims to develop, on the one hand, the central postulates of Sommer's proposal and then confront them in the light of criticism and to what extent such criticism is central. The foregoing gives light of a discussion that more than leaning to the controversy shows the nutrient of a fruitful investigation.
\end{abstract}

Keywords: Nietzsche, Sommer, experimental philosophy, Zarathustra.

Fecha de recepción: 1 de junio de 2019

Fecha de aceptación: 21 de agosto de 2019

Forma de citar (APA): Choque, O. (2020). El pensador bajo la máscara. Aporías a la filosofía experimental. Revista Filosofía UIS, 19(2), https://doi.org/10.18273/revfil.v19n2-2020002

Forma de citar (Harvard): Choque, O. (2020). El pensador bajo la máscara. Aporías a la filosofía experimental. Revista Filosofía UIS, 19(2), 21-34.

Osman Daniel Choque Aliaga: boliviano. Magíster en Filosofía Contemporánea de la Universidad de San Buenaventura, Colombia. Estudiante de doctorado en Albert-Ludwigs-Universität Freiburg, Alemania.

ORCID iD: https://orcid.org/0000-0003-4374-8708

Correo electrónico: junker.odca@gmail.com; ochoque@academia.usbbog.edu.co

"Artículo de investigación científica.

Revista Filosofía UIS, vol. 19 n. ${ }^{\circ}$ 2, julio - diciembre 2020 


\section{El pensador bajo la máscara. Aporías a la filosofía experimental}

\section{Introducción}

Es un recurso frecuente que entre las opiniones del gremio de la filosofía estas se inclinen a considerar la figura preeminente de Heidegger en relación a Nietzsche. La cita de que "[...] la filosofía de Nietzsche es metafísica y toda metafísica es platonismo" (Heidegger, 2013, p. 22) ha deambulado por el murmullo de los intérpretes que se inclinan más por la figura del filósofo de Meßkirch que por la del filósofo de Röcken. Esa afirmación podría quizá considerarse con cierta discrepancia y plantear algunas interesantes consideraciones acerca de la interpretación de Heidegger en la figura de Nietzsche. ¿Fue realmente Heidegger un verdadero intérprete de Nietzsche? ¿Cómo puede situarse dicha interpretación?

Heidegger publica en el año de 1961 el texto Nietzsche en dos tomos. Una interpretación pormenorizada de las ideas centrales de la filosofía de Nietzsche. El término de "ideas centrales" debe considerarse, sin embargo, con precaución. Hasta inicios del siglo XXI la interpretación acerca de Nietzsche podía condensarse en tres apartados: "El eterno retorno", "La voluntad de poder" y el "Superhombre". De tal manera que quien entendía dichos conceptos estaba encarrilado para acercarse a una comprensión más o menos "correcta" del filósofo alemán. Hoy por hoy ese camino se ha enfrentado con la investigación reciente en Alemania, pero de esto hablaremos más adelante. Volviendo a la interpretación heideggeriana de Nietzsche el juicio que debe darse a dicha interpretación es, digámoslo de una vez por todas, "sistemáticamente abusiva". La razón que nos lleva a dicha afirmación radica en que Heidegger no conoció la edición crítica de las obras de Nietzsche, es decir, los comentarios que Giorgio Colli y Mazzino Montinari (KGW y KGB) hicieron de la obra nietzscheana y fundamentalmente del lugar que debe darse a la obra La voluntad de poder. Esa ausencia de la edición crítica llevó a Heidegger a leer, bajo sus propios intereses filosóficos, de manera arbitraria la obra de Nietzsche. Dentro de la publicación de Heidegger se halla el eco que expresa que el uso de los fragmentos póstumos, Der Nachlass, es determinante en la interpretación nietzscheana. Heidegger dirá al respecto: "Dentro de los Nachlass está presente la verdadera filosofía de Nietzsche" (Heidegger, 2013, p. 220). Ciertamente, la presencia e importancia de la interpretación heideggeriana 
de Nietzsche tiene un valor importante en cuanto forma ontogenética, es decir, en cuanto al proceso de la recepción filosófica de Nietzsche y no en su aspecto filogenético, como lo describe Marcuse en su obra Eros y civilización en perspectiva freudiana, en miras a una posible final y definitiva interpretación nietzscheana (cfr. Marcuse, 2010, p. 39). La interpretación acerca de Nietzsche se inclinó al campo de la política y luego al de la literatura; Heidegger fue, entre otros aspectos, quien resaltó los postulados filosóficos de su pensamiento.

El meollo de la cuestión que gira entre las interpretaciones literarias y las tendencias políticas, fue superado por Heidegger quien dio a Nietzsche un lugar dentro de la filosofía, es decir, desde la publicación de Heidegger sobre Nietzsche, el filósofo de Röcken fue considerado con el título específico de filósofo. (Choque, 2018a, p. 21)

En esta línea se nos ofrece la publicación de Andreas Urs Sommer, quien sostiene que la interpretación heideggeriana ha sido sobrevalorada y que lo más adecuado para el mundo filosófico es una postura un poco más reservada ${ }^{1}$. En ese sentido, un camino por el que los actuales intérpretes de Nietzsche se inclinan consiste en posar la mirada en el filósofo de Röcken desde una perspectiva nueva y fresca, en la que podamos alejarnos de la "vieja" interpretación de Nietzsche y comprender, de esa manera, la importancia de su pensamiento en el presente ${ }^{2}$. Por su parte, en esa línea interpretativa se viene llevando a cabo el trabajo por la Heidelberger Akademie der Wissenschaften con la publicación de Nietzsche Kommentar. Recientemente, Sommer publicó su obra Nietzsche und die Folgen donde puntualiza las implicaciones de la nueva interpretación de Nietzsche y, por otro lado, es en esa obra donde está presente el uso de la filosofía experimental, una cuestión en la que nos detendremos a continuación.

Para desarrollar los intereses de este trabajo, en primer lugar, nos detendremos en detallar las ideas principales de lo que Sommer denominó filosofía experimental; en segundo lugar, desglosaremos las preocupaciones de Jordi Morillas (2019) en su texto "Consideraciones acerca de la filosofía experimental de Friedrich Nietzsche. A propósito de la obra Nietzsche und die Folgen de Andreas Urs Sommer" para quien dicha filosofía experimental podría ir en contra vía de las opiniones mismas de Nietzsche. En tercer y último lugar, ofreceremos elementos que podrán dar cuenta que la filosofía experimental no se aleja de las opiniones fundamentales de Nietzsche, sino que las ahonda. Todo lo anterior mostrará la riqueza de una discusión que va creciendo en los ambientes académicos.

\footnotetext{
${ }^{1}$ cfr. Sommer, 2017b; Heidegger, 2013.

2 cfr. Sommer, 2017a; Schwab, 2015; Tongeren, 1999; Babich, 2010.
} 


\section{Los experimentos de la filosofía nietzscheana}

¿Qué es la Experimentalphilosophie? Como se verá a continuación, en principio este término no aparece en la obra de Sommer Nietzsche und die Folgen (2017a), sino que se encuentra en otro trabajo. La filosofía experimental también está presente en un artículo suyo titulado: "'Dios ha muerto' y '¿Dionisio contra el crucificado?'” (2006). Sin embargo, en ese trabajo Sommer considera otra cuestión paralela que va de la mano de lo que se ha explicado: el escepticismo experimental, noción que sirve para entender la filosofía experimental. Por lo anterior, en primer lugar, es oportuno dar una rápida mirada a dicho concepto y hacer una explicación a partir de los textos de Nietzsche.

De manera casi generalizada, la interpretación convencional de Nietzsche se inclinó a afirmar que todas las ideas filosóficas del pensador alemán exigen del lector una puesta en escena, un compromiso con la vida, una postura crítica dirigida especialmente a la religión y la supremacía de un cierto tipo de voluntad que podría doblegar las decisiones pequeñas. De la misma manera, varias interpretaciones han querido fundar un tipo de protohistoria a raíz de los enunciados de Nietzsche ${ }^{3}$. No se puede dejar de lado y no dar el debido merecimiento a las interpretaciones de diversos autores, tan valiosas en su momento, que han marcado la historia de la recepción del pensador alemán, y que a la vez han contribuido de manera notable a la comprensión de su filosofía. (Fink, 2017; Muller-Lauter, 1974). No obstante, el presente obliga a considerar de otro modo las ideas de Nietzsche. Dentro de las interpretaciones actuales se encuentran la filosofía experimental de Sommer. En el texto "'Dios ha muerto' y '¿Dionisio contra el crucificado?'”, Sommer afirma que a raíz de la gran cantidad de artificios literarios presentes en la obra de Nietzsche, como lo demuestran los escritos Así habló Zaratustra y La gaya ciencia, se abre una distancia entre las opiniones de Nietzsche y el contenido de los artificios literarios. Dice Sommer (2006) al respecto:

La creación de tales artificios literarios es un componente integral de la filosofía experimental de Nietzsche. Estas figuras artificiales, tal y como Nietzsche las utiliza, son comodines ideales de la filosofía experimental in praxi: su actuar y su hablar eximen al filósofo experimental de tener que hacer referencia a una misma posición o a comprometerse con una doctrina u opinión cualquiera. (p. 49)

En ese sentido, los artificios literarios de la obra nietzscheana abrirían el paso para la filosofía experimental y, sobre todo, introducirían el inicio de unos presupuestos que desprenden toda responsabilidad filosófica de lo que el filósofo de Röcken afirmó y defendió. "La creación de tales artificios literarios es un componente integral de la filosofía experimental de Nietzsche" (Sommer, 2006, 
p. 49). Sommer afirma que dicha filosofía experimental esgrime en el escritor las opiniones en aquello que escribe, es decir, el autor no pretende decir lo que piensa ad litteram sino recrear una posible situación en el lector. Dice Choque (2018a):

La "filosofía experimental" lejos de querer explicar las ideas del autor del texto lo que pretende es evitar al lector que caiga en dicha situación. Este artificio literario separa al autor de sus textos $y$, de esa manera, de una directa interpretación. La tarea del filósofo, que en este caso es Nietzsche, es provocar en el lector una situación a partir de lo que él relata. (p. 18)

En definitiva, la filosofía experimental, tal como lo plantea Sommer, esgrime las posiciones filosóficas del autor, evitando de esa manera una relación directa con aquello que él pretende mencionar, en definitiva, "delegar la decisión de lo que es verdadero e importante al lector" (2006, p. 50). En Nietzsche und die Folgen, Sommer continúa y estudia el concepto que se ha venido tratando y describe un matiz importante, esta vez haciendo un tipo de juego a partir del uso de la escritura. En ocasiones, según Sommer, Nietzsche juega en sus escritos con el "yo" y el "nosotros", la primera persona pertenecería a la pretensión directa de manifestar una idea, como pensador; y la segunda forma, el "nosotros", hace copartícipe del relato al auditorio. En esto radicaría la intención del filósofo alemán para transmitir sus ideas, pues generalmente las ideas más audaces de Nietzsche están formuladas a partir del "nosotros". Dice Sommer: "Con estos "nosotros" se roba el filósofo experimental de nuevo un poco la responsabilidad de su escritura: se puede decir que él es como "yo" (eso no sucede aquí) cuando el "nosotros" se pone a hacer grandes obras" (2017a, p. 49) ${ }^{4}$. La filosofía experimental no expresa el "yo" en sus relatos, sino que se inclina a esconderse, de manera irónica, en el uso del "nosotros".

Para argumentar lo anterior nos serviremos del parágrafo 125 de La gaya ciencia. Este texto asume un rol importante frente a otras publicaciones de Nietzsche. Efectivamente, no "es posible encontrar en los textos de Nietzsche otro parágrafo o aforismo que haya llamado la atención y a la vez provocado la confusión de un gran número de lectores" (Choque, 2019, p. 140). En el parágrafo 125 de La gaya ciencia es donde se encuentra la sentencia "Dios ha muerto". Adentrándonos en el texto, encontramos la presencia de un personaje, el loco, quien tiene un anuncio y lo comunica a la gente del mercado. Aunque no se encuentra dentro de nuestros intereses el pormenorizar valiosos detalles en ese texto (cfr. Sommer, 2006; Choque, 2019 y 2018a), podemos solo tomar una parte del relato que iluminará el sentido del "yo" y el "nosotros". El texto comienza de la siguiente manera: "No habéis oído hablar de ese hombre loco" (Nietzsche, 2007, p. 114).

$\overline{{ }^{4} \text { La traducción es propia. }}$ 
[eKGWB/FW-125] ${ }^{5}$. A partir de lo anterior, no es Nietzsche quien se manifiesta como "yo os menciono" o "quiero plantearles tal cuestión", sino que pone en palabras de otra persona su mensaje, por un lado, y, por otro, este mensaje es compartido hacia los otros, la "gente del mercado". Sommer (2006) plantea que: "el diagnóstico de la muerte de Dios misma no está asociado a ningún sujeto; nadie se hace responsable de ella" (p. 51). ¿Por qué Nietzsche no usa el "yo" para este importante mensaje? En ello radica, para Sommer, el cuidadoso uso de un juego intelectual en los lectores, es decir, Nietzsche pretende despertar en los lectores un tipo de juego mental a partir de los artificios literarios empleados en su obra.

El diagnóstico de la muerte de Dios misma no está asociado a ningún sujeto; nadie se hace responsable de ella. En cada texto normal de prosa filosófica uno creería reconocer en él muy fácilmente la opinión de los autores; en el caso de un escrito de Nietzsche, que aplica virtuosamente todos los artificios para el encubrimiento de las verdaderas opiniones de su autor, se presta más atención. (Sommer, 2006, p. 51)

Ahora bien, como se menciona líneas arriba, la filosofía experimental va de la mano de lo que Sommer denominó escepticismo experimental. ¿De qué manera debemos usar el escepticismo experimental a la luz de la filosofía experimental? En primer lugar, necesitamos determinar en qué medida un tipo de escepticismo puede considerarse como el portón para acercarse a la filosofía experimental, es decir, debemos ponernos en la posición del loco. Retomando la figura del loco que se describe en el parágrafo 125 de La gaya ciencia, este se expresa de la siguiente manera: "«iA dónde ha ido Dios?», gritó, «iyo os lo voy a decir! iNosotros lo hemos matado —vosotros y yo! iTodos nosotros somos sus asesinos!»" (Nietzsche, 2007, p. 115). [eKGWB/FW-125]. El loco sabe que "Dios ha muerto" y su tarea radica en llevar el mensaje a la gente del mercado. Es decir, el loco tiene la intención de llevar el mensaje y ese actuar busca obtener algo "nuevo" al dar la noticia. Tiene una intención comunicativa y en ello busca obtener algo nuevo. En lo anterior, el escepticismo encontrado es la puesta en escena para obtener algo nuevo de lo que antes no se tenía. El término escepticismo, dentro de la tradición filosófica, puede entenderse como una doctrina que no acepta una verdad definitiva. Pero a la luz de lo que Sommer plantea al interpretar el concepto de escepticismo experimental, el lector debe asumir una posición, aunque sea de

\footnotetext{
${ }^{5}$ Las referencias remiten a la traducción del castellano y al original en alemán de la obra de Nietzsche. Luego de la citación como aparece en la bibliografía, se ha puesto en corchetes la citación del texto en alemán tal como aparece en la http://www.nietzschesource.org/\#eKGWB. Primero va la abreviatura eKGWB, luego el nombre del texto y el número a que se hace referencia como se puede encontrar en la página antes mencionada. "La edición crítica digital de las obras y la correspondencia de Nietzsche al cuidado de Paolo D'lorio y publicada por la Nietzsche Source (eKGWB) se basa en el texto crítico establecido por Giorgio Colli y Mazzino Montinari (KGW y KGB). La calidad de esta edición se basa en un riguroso trabajo de transcripción del texto de la edición impresa a la edición digital" (D’lorio, s.f.).
} 
manera provisional. Dicha actitud es la máxima del escepticismo experimental de Nietzsche en cuanto que es el inicio de un proceso creador. Dirá Sommer (2006): "el escepticismo es entonces una filosofía experimental creadora" (p. 63). El loco, como vimos, sabe que "Dios ha muerto" pero él busca aferrarse del mensaje con el fin de transmitirlo, y espera un efecto creador a raíz de dicho acto. Por ello, la "muerte de Dios", para él, no es otra cosa que un profundo momento creador. "La condición escéptica libera al sujeto de lo que considera hasta ese momento como valedero y lo posibilita para crear algo nuevo" (Choque, 2018a, p. 10).

Por lo tanto, si la filosofía experimental exime la responsabilidad del autor en cuanto transmisor de sus ideas, da paso a libres interpretaciones por parte de los lectores; estos, en ese sentido, hacen de testigos y es sobre quienes cae el juego de imaginar las consecuencias de lo dicho por el autor. El escepticismo experimental ayudará a comprender la filosofía experimental mientras provoque en el lector la sospecha, la desconfianza ante lo que él consideraba como cierto en las palabras del autor. Ese proceso efectivamente requiere de facultades creativas; el escepticismo experimental sugiere contar con una actitud creadora y la creatividad va de la mano de la filosofía experimental. Ambas cuestiones que venimos estudiando van enfocadas hacia un tipo de ejercicio mental en donde el lector deberá enfrentarse a posibles eventualidades y se pretende que ello haga nacer en su mente un proceso de ideas nuevas. En primer lugar, el escepticismo experimental lleva al lector a sospechar de las verdades. En un segundo momento, la filosofía experimental exime al autor del texto de las ideas presentadas. El lector deja de considerar las ideas del autor como directas y asume una actitud de espectador en cuanto imagina las consecuencias de lo que dicho autor pretendió decir. El loco y Zaratustra, por ejemplo, son ejercicios personificados que no representan las propias ideas de Nietzsche. Hasta este momento, la propuesta de Sommer muestra a un Nietzsche distinto a como antaño se lo comprendía, en definitiva, una nueva interpretación en torno a Nietzsche a partir de la filosofía experimental. Sin embargo, la crítica ha puesto su mirada en una cuestión elemental: si dicha filosofía experimental explica, y de qué manera, lo que Nietzsche pretendía que fuese su legado. Para desarrollar lo anterior se tomará como guía el trabajo de Morillas (2019).

\section{Consideraciones acerca de la filosofía experimental}

El profesor de la Universidad de Lübeck, Jordi Morillas, publicó un texto titulado "Consideraciones acerca de la filosofía experimental de Friedrich Nietzsche. A propósito de la obra Nietzsche und die Folgen de Andreas Urs Sommer" (2019) en el que pretende poner en cuestión los puntos débiles de la interpretación que hace Sommer de Nietzsche, basándose en los textos que el profesor de la Universidad de Friburgo publicó acerca de Nietzsche.

El primer argumento que pone sobre la mesa Morillas es establecer que 
efectivamente en la interpretación de Sommer se encuentra una importante empresa hermenéutica. Haciendo referencia, por un lado, al importante proyecto que tiene por nombre Nietzsche Kommentar, dirigido por la Heidelberger Akademie der Wissenschaften, el texto que Morillas se detiene a comentar es la publicación Nietzsche und die Folgen, realizada por Sommer. La siguiente frase, según Morillas, condensa de manera medular el sentido de la filosofía experimental: "Nietzsche es un filósofo que utilizó las doctrinas como experimentos intelectuales y existenciales. Su filosofía no es ninguna doctrina, sino un hacer, una praxis de transformación pensante del mundo" (Sommer, 2017a, p. 2) ${ }^{6}$. Por otro lado, Morillas se detiene en la relación de Nietzsche con los personajes que aparecen en varios de sus escritos, de manera especial en el libro titulado Así habló Zaratustra,

Por lo que se refiere a las obras filosóficas de Nietzsche, Sommer defiende la tesis de que no hay que identificar al héroe de Así habló Zaratustra, quien se presenta como un maestro sin éxito (pág. 54), con Nietzsche (págs. 53 y 61), sosteniendo que este escrito "no es ninguna novela de tesis que ponga a la venta al público teorías filosóficas en un envoltorio escasamente poético". (Morillas, 2019, p. 208)

Por lo tanto, habría una distancia entre Zaratustra y el propio Nietzsche. Sommer recomienda tener cuidado a la hora de relacionar al autor de los textos con las figuras que se hallan en sus escritos. No sería pertinente, en ese sentido, poner en boca de Nietzsche las frases, las reflexiones, las ideas de Zaratustra. ¿Cuál es la tarea entonces de dichos relatos? ¿Qué pretenden? Nietzsche, según Morillas, pretendería llevar al lector a un terreno en donde se exija un trabajo de reflexión, un tipo de laboratorio de pensamiento en donde se elabore "un intento de hacer 'experimentos' a nivel intelectual" (2019, p. 208). Morillas considera que si toda la obra de Nietzsche puede ser juzgada a la luz de la filosofía experimental no podríamos saber a ciencia cierta cuáles son realmente las ideas "verdaderas" de Nietzsche, pues todos los escritos del filósofo alemán podrían eximir al autor de lo que realmente quiso decir.

En concreto, se ha mencionado el recurso al que se inclinaron los intérpretes de Nietzsche, ahora bien, se toma como ejemplo "El eterno retorno", que no tendría otra tarea fundamental que hacer de juegos experimentales. Por otro lado, como lo deja ver Morillas, Sommer también deja de lado la interpretación heideggeriana que tanto cotizaba el uso de los Nachlass, pues también esos escritos formarían parte de un "campo experimental" (Sommer, 2017a, p. 101). Hasta aquí, el desarrollo que sigue Morillas es bastante positivo; sin embargo, en las siguientes páginas no tarda en iniciar la crítica a la propuesta del pensador suizo: "El resultado inmediato de una lectura semejante es una relativización y debilitación del pensamiento de Nietzsche, con la consecuente pérdida de toda autonomía, originalidad e importancia histórica" (Morillas, 2019, p. 210). Para

${ }^{6}$ Traducción realizada por Morillas. 
Morillas, la propuesta de Sommer inevitablemente llevaría a la "relativización" y a que la filosofía de Nietzsche pierda toda "originalidad e importancia histórica". ¿Sería realmente ese el destino casi inevitable de la filosofía experimental? Morillas (2019) culmina su texto con las siguientes palabras:

[...] conlleva el intrínseco peligro de despreciarlo como pensador. Pues, ¿qué queda de un filósofo al cual se le despoja de sus doctrinas filosóficas principales? La ironía y el campo libre para todo tipo de interpretaciones "pluralistas" y "multiperspectivistas". ¿No iría semejante lectura en contra de lo reivindicado por el propio filósofo?, ya que, ¿̇uál era la finalidad de su último escrito, Ecce homo, sino combatir avant la lettre todo este tipo de interpretaciones de su persona y de su pensamiento a la vez que reivindicar la importancia y la seriedad histórica de su proyecto filosófico? (p. 213)

En suma, Morillas explica que, en primer lugar, la filosofía experimental negaría todo signo de originalidad en Nietzsche y terminaría fundamentalmente quitándole el título de "pensador", puesto que no se podría percibir con el valor que merecen sus ideas filosóficas. En segundo lugar, que la filosofía experimental no pertenecería a lo que el autor quiso, es decir, no formaría parte del legado de las opiniones del pensador y de su futuro legado. ¿Es cierto lo anterior? ¿No será otra forma de interpretación nietzscheana tan parecida a la del siglo XX? ¿No es acaso volver a usar "vino viejo en tinajas viejas"?

La manera más efectiva de poder encontrar algunos caminos y luces a los planteamientos de Morillas sobre la interpretación de Sommer es mediante la propia obra de Nietzsche. Ese será el camino que se llevará a continuación.

\section{El Crepúsculo de los ídolos}

El escrito Crepúsculo de los ídolos fue publicado en 1887, un par de años antes de que Nietzsche iniciara su "derrumbe". Al parecer la interpretación tradicional que se ha hecho del pensador alemán no ha tomado en serio esta obra comparada con otras obras, que son fruto de la producción intelectual del autor, ya que el juicio que se dictó sobre este texto se enmarca en unas posibles inconsistencias a raíz de su "locura" cada vez en aumento en esa época.

Sommer piensa que el Crepúsculo de los ídolos, obra gemela del Anticristo, lejos de ocupar el puesto que la crítica le ha dado, posee un lugar importante en la producción de Nietzsche ${ }^{7}$. No se trata de un texto que describe los inicios de una falta de "cordura" o "lucidez" sino que se está ante un texto "central en la obra de Nietzsche, porque comprime y compendia el pensamiento tardío y ofrece una síntesis y un panorama general sobre las del autor" (Sommer en De Santiago Guervós, s.f., p. 1). Para ahondar en la propuesta de Morillas, se considera el

${ }^{7}$ cfr. De Santiago Guervós, s.f. 
Crepúsculo de los ídolos como un texto valioso para la interpretación del autor, pues como se mencionó, ofrece un compendio de su pensamiento.

En el Crepúsculo de los ídolos se encuentra un texto titulado "La razón en la filosofía". En este, dice el filósofo de Röcken:

Los filósofos creen otorgar un honor a una cosa cuando la deshistorizan, sub specie aeterni (desde la perspectiva de lo eterno) cuando hacen de ella una momia. Todo lo que han estado utilizando los filósofos desde hace miles de años no son más que momias conceptuales; nada real ha salido con vida de sus manos. Cuando esos idólatras adoran algo, lo matan y lo disecan. iQué mortalmente peligrosos resultan cuando adoran! Para ellos, la muerte, el cambio, la vejez, al igual que la fecundación y el desarrollo constituyen objeciones, e incluso refutaciones. Lo que es, no deviene; lo que deviene, no es ... Ahora bien, todos ellos creen, incluso de una forma desesperada, en lo que es. Pero como no pueden apoderarse de lo que es, tratan de explicar por qué se les resiste. (Nietzsche, 2002, p. 51) [eKGWB/ GD-Vernunft-1]

¿A qué se refería Nietzsche con "momias conceptuales"? ¿Qué significa que "todos ellos creen, incluso de una forma desesperada, en lo que es"? Ciertamente, Nietzsche no posee una simpatía con aquellos que pretenden encasillar las ideas en conceptos, en reducir los pensamientos a expresiones finales y últimas. En ese sentido, el filósofo alemán desea alejarse de los conceptos, de las inclinaciones que consideran todo como expresiones últimas, y este mismo criterio puede aplicarse a su propia filosofía. Por lo tanto, el acercamiento que se haga a su obra no debe pretender hallar en esta un pensamiento en sí mismo terminado y concluido. Dice Nietzsche (2002) en uno de sus escritos: "Los hombres póstumos - yo, por ejemplo- son peor comprendidos que los tempestivos, pero mejor oídos. Dicho con más rigor: no somos comprendidos jamás - y de ahí nuestra autoridad" (p. 7). [eKGWB/GD-Vernunft-15]. ¿Afirmar que Nietzsche solo puede ser entendido de una forma, a la vez "única y definitiva" no nos llevaría a la postura de las "momias conceptuales"? Si tomamos con la debida seriedad el texto, Nietzsche no estaría de acuerdo en que se le atribuya que su filosofía "es" de ese modo, es decir, el pensador alemán podría recordar la actitud de los filósofos que creen desesperadamente en lo que "es".

Pero, ¿qué "es" la filosofía de Nietzsche? Es una pregunta que, a partir de la cita anterior, no debe ser la primera en ser respondida. "La razón en la filosofía" es un texto que sin lugar a dudas puede dar luces para comprender la postura de Morillas y considerar que las supuestas "doctrinas filosóficas principales" de Nietzsche, en el caso de que hoy puedan considerarse como tales, necesitan quizá tomar asiento. Sin embargo, la interpretación nietzscheana ha recurrido al argumento que considera la existencia de "doctrinas filosóficas principales" en Nietzsche. ¿No podría considerarse lo anterior como una trinchera difícil 
de franquear? ¿Realmente quiso Nietzsche que sus ideas filosóficas fuesen consideradas dentro de su pensamiento como "las principales" separadas de las que no lo son? En efecto, es una pregunta que amerita la paciente, dedicada y atenta respuesta.

\section{Conclusión}

A medida que se ha desarrollado la filosofía experimental como un nuevo camino para comprender las ideas de Nietzsche, se ha descrito que esa es la línea de interpretación que se está trabajando en Alemania. En ese sentido, se hacen oportunos los comentarios de Morillas acerca de la propuesta de Sommer, una proposición que puede crear varias opiniones en cuanto a su posible aceptación. Sin embargo, en medio de todo hace falta un poco de ironía a la hora de acercarse al pensamiento de Nietzsche. Un tipo de ironía que es inseparable de la manera como se conciben sus ideas filosóficas ${ }^{8}$.

Ahora bien, no se puede dejar de lado el aporte de la filosofía experimental, puesto que esta propuesta lleva al lector a un terreno en donde este debe dar de sí un esfuerzo intelectual. El caso de la "muerte de Dios" que figura en boca del loco, obliga al lector, como se ha mencionado, a no imaginar un hecho real y determinante sino a que este pueda imaginar las consecuencias y efectos de dicha muerte. Ese efecto, inevitablemente, evoca en el lector una apertura a la imaginación en donde el ejercicio intelectual, la voluntad de pensar, el proceso que requiere poner en escena al pensador, se hace presente.

Según Sommer, una de las importantes tareas de Nietzsche fue que el lector no lo identificara con Zaratustra, sino que el lector pudiera imaginar, crear posibles escenas. No estaba lejos Sloterdijk cuando dijo que Nietzsche "es el guía en la bella tentación de crear figuras de vida teniendo como materiales el talento y el carácter" (2010, p. 113). Fundamentalmente, Morillas olvida que la lectura de Nietzsche exige del lector un esfuerzo intelectual, que implica comprender las posibilidades de los enunciados y que lleva a considera que lo que piensa y cree es susceptible de error.

El futuro de las investigaciones sobre Nietzsche, como de todo pensador, necesita ese espacio donde se dejen reposar, por el momento, los trabajos que los intérpretes han elaborado y dar el paso necesario para un escenario ulterior. No se conoce, ni se tiene una idea clara para saber si este camino, el de la filosofía experimental, es el más adecuado o si, por el contrario, se va en contra de las profundas voluntades del pensador. Solo una investigación paciente y documentalista, y el avatar de los sucesos posteriores podrán realmente dar las

${ }^{8} \mathrm{cfr}$. Sommer, 2017a, pp. 182-190. 
respuestas pertinentes.

\section{Referencias}

Babich, B. (2010). "Un problema con cuernos... el problema de la ciencia misma". La crítica nietzscheana a la razón científica. (L. E. De Santiago Guervós, trad.). Estudios Nietzsche, 8, 14-53.

Botero, D. (2002). La voluntad de poder de Nietzsche. Unibiblos.

Choque, O. (2018a). El filósofo y su filosofía. La 'filosofía experimental' de Nietzsche. Fragmentos de filosofía, 16, 1-21.

Choque, O. (2018b). Ciencia y creatividad en Friedrich Nietzsche. Con-Sciencias sociales, 19, 20-31.

Choque, O. (2019). "Dios ha muerto" y la cuestión de la ciencia en Nietzsche. Estudios de Filosofía, 59, 139-166.

De Santiago Guervós, L. E. (Sin fecha). Sommer [Reseña]. www.uma.es/ nietzscheseden/espanol/recensiones/SOMMER-14.pdf

D'lorio, P. (Sin fecha). La edición crítica digital de las obras y la correspondencia de Nietzsche. www.uma.es/nietzsche-seden/obra/eKGWB.pdf

Fink, E. (2017). La filosofía de Nietzsche. Alianza Editorial.

Heidegger, M. (2013). Nietzsche. Ariel.

Marcuse, H. (2010). Eros y civilización. Ariel.

Morillas, J. (2019). Consideraciones acerca de la filosofía experimental de Friedrich Nietzsche. A propósito de la obra Nietzsche und die Folgen de Andreas Urs Sommer. Daimon, 76, 207-214. Http://doi.org/10.6018/daimon/325351

Muller-Lauter, W. (1974). Nietzsches Lehre vom Willen zur Macht. NietzscheStudium, 3, 1-60.

Nietzsche, F. (2002). Crepúsculo de los ídolos. O Cómo se filosofa con el Martillo. Alianza Editorial.

Nietzsche, F. (2007). La gaya ciencia. (A. Mardomingo, trad.). Edaf.

Schwab, P. (2015). "Critique of 'the System' and Experimental Philosophy 
- Nietzsche and Kierkegaard". In K. Dawn Hay \& L. R. dos Santos. (Eds.), Nietzsche, German Idealism and Its Critics (pp. 223-245). De Gruyter.

Sloterdijk, P. (2010). Temperamentos filosóficos. De Platón a Foucault. Siruela.

Sommer, A. U. (2006). «Dios ha muerto» y «¿Dionisio contra el crucificado?» Sobre la crítica de Nietzsche a la religión y al cristianismo. (L. E. De Santiago Guervós, trad.). Estudios Nietzsche, 6, 47-64.

Sommer, A. U. (2017a). Nietzsche und die Folgen. J. B. Metzler.

Sommer, A. U. (2017b). Nietzsche como placa giratória "na" modernidade? O Nietzsche de Heidegger nos Cadernos negros e o papel do filósofo. En E. Nasser e L. Rubira (org.). Nietzsche no século XXI (pp. 263-287). Editora Zouk.

Tongeren, P. V. (1999). Reinterpreting Modern Culture: An introduction to Nietzsche's philosophy. West Lafayette. 